\title{
AN INDUCTION BASED ON A HYBRID OF DRSA AND DEMATEL FOR ANALYZING COMPETITIVENESS 2012
}

\author{
Yu-Chien $\mathrm{Ko}^{1}$, Chao Hsien-Wen ${ }^{1}$ \\ Department of Information Management \\ Chung Hua University \\ Hsinchu, Taiwan \\ E-mail: eugene@chu.edu.tw; E-mail: e09910013@chu.edu.tw \\ Gwo-Hshiung Tzeng ${ }^{2,3}$ \\ ${ }^{2}$ Graduate Institute of Innovation and Project Management, Kainan University; No. 1 Kainan Road, \\ Luchu, Taoyuan County 338, Taiwan \\ ${ }^{3}$ Institute of Management of Technology, National Chiao Tung University \\ 1001 Ta-Hsueh Road, Hsinchu 300, Taiwan \\ E-mail: ghtzeng@mail.knu.edu.tw; ghtzeng@cc.nctu.edu.tw
}

\begin{abstract}
Faced with the worldwide challenges, the interdependence among criteria deserves paying attention for national competitiveness. When considering the influence in others and the sensitivity to others, this issue becomes complicated and hard. In this research, a hybrid of DRSA and DEMATEL is proposed to generate an interdependence map by the induction information, which helps in understanding the inside of competitiveness. For an empirical illustration, the proposed method is applied to analyze on World Competitiveness Yearbook 2012. The result shows that institutional framework, health and environment, and basic infrastructure are significant to other criteria.
\end{abstract}

Keywords: competitiveness, dominance-based rough set approach (DRSA), Decision-Making Trial and Evaluation Laboratory (DEMATEL)

\section{Introduction}

Competitiveness has been playing an aggregated power of a nation to enhance people's lives and cope with worldly challenges (U.S. President's Commission on Industrial Competitiveness, 1985; WEF, 2003; IMD, 2012). National leaders need pay careful attention not only to competition but also to interdependence among criteria which can help and enhance policy-making. There are two attractive points in the criteria interdependence. One is the influence of a criterion in others. The other is the sensitivity of a criterion to others. This research proposes a network relationship map (NRM) of criteria interdependence based on a hybrid of DRSA and DEMATEL (HDD). The hybrid can present the knowledge of influence and sensitivity among criteria by induction rules. Empirically, the proposed method is applied on the World Competitiveness Yearbook (WCY) in 2012. The result reveals significant criteria with the influence and sensitivity in NRM.

The remainder of this paper is organized by reviewing national competitiveness, DRSA, and DecisionMaking Trial and Evaluation Laboratory (DEMATEL) in Section 2, proposing the hybrid method in Section 3, describing the application results in Section 4, and discussing and concluding the paper in Section 5.

\section{Literature review}

DRSA is a powerful technique of relational structure and has been successfully applied in many fields (Greco et al. 2000, 2001, 2002). In classification application, it can be used to induce objects assigned to 
$C l_{t}^{2}$ (the upper ward union classes which include objects ranked at least $t^{\text {th }}$ ) or to $\mathrm{Cl}_{t}^{<}$(the downward union of classes which includes objects ranked less than $t^{\text {th }}$ ), where $\mathrm{Cl}$ is a cluster set containing ordered classes $C l_{t}, t \in T$ and $T=\{1,2, \ldots, n\}$. The formulations for the above statement can be expressed as $C l=\left\{C l_{1}, \ldots, C l_{t}, \ldots, C l_{n}\right\}, C l_{1}=\{y \in U: y$ is ranked in the top position $\}, C l_{2}=\{y \in U: y$ is ranked in the second position $\}, \ldots$, and $C l_{n}=\{y \in U: y$ is ranked in the bottom position $\}$ where $U$ is a set with decision makers' preference orders. For all $s, t \in T$ and $s \geq t$ (rank of $s \geq \operatorname{rank}$ of $t$ ), every object in $C l_{s}$ is preferred to be at least as good as any of object in $C l_{t}$. They are constructed as:

The dominating union: $C l_{t}^{\geq}=\bigcup_{s \geq t} C l_{s}$ for $s \geq t$; the dominated union: $C l_{t}^{<}=\bigcup_{s<t} C l_{s}$ for $s<t$.

Another representation of the dominating set relies on a set of criteria, $P$. It follows the dominance principle of requiring each chosen object at least as good as a boundary object $x$ in all considered criteria. The granules of a dominating set based on $P$ can be viewed as the granular cones in the criteria value space. Vice versa the dominated sets follow the dominance principle and have granules in the opposite direction. These cones are categorized into $P$-dominating and $P$-dominated sets [26], respectively. It is said that object $y P$-dominates object $x$ with respect to a criteria set $P$ (denotation $y D_{P} x$ ). Given $x, y \in U$ and $P$, let dominance sets as:

$P$-dominating set: $D_{P}^{+}(x)=\left\{y \in U, y D_{P} x\right\}$ and $P$-dominated set: $D_{P}^{-}(x)=\left\{y \in U, x D_{P} y\right\}$ where $x, y \in C l$, $x$ plays a role for the boundary of $D_{P}^{+}(x)$ or $D_{P}^{-}(x), y \succsim_{\approx q} x$ for $D_{P}^{+}(x), x \succsim_{\sim q} y$ for $D_{P}^{-}(x)$, and all $q \in P$. Two approximations are defined for illustrating the dominance consistency. The association between $C l_{t}^{\geq}$ and $P$-dominating set should keep dominance consistency requiring $y \in C l_{t}^{\geq}$and $y \in P$-dominating.

$$
\begin{aligned}
& \underline{P}\left(C l_{t}^{\geq}\right)=\left\{x \in C l_{t}^{\geq}, D_{P}^{+}(x) \subseteq C l_{t}^{\geq}\right\}, \bar{P}\left(C l_{t}^{\geq}\right)=\bigcup_{x \in C l_{t}^{\Sigma^{<}}} D_{P}^{+}(x), \operatorname{Bnp}\left(C l_{t}^{\geq}\right)=\bar{P}\left(C l_{t}^{\geq}\right)-\underline{P}\left(C l_{t}^{\geq}\right) \\
& \underline{P}\left(C l_{t}^{<}\right)=\left\{x \in C l_{t}^{<}, D_{P}^{-}(x) \subseteq C l_{t}^{<}\right\}, \bar{P}\left(C l_{t}^{<}\right)=\bigcup_{x \in C l_{t}^{<}} D_{P}^{-}(x), B n p\left(C l_{t}^{<}\right)=\bar{P}\left(C l_{t}^{<}\right)-\underline{P}\left(C l_{t}^{<}\right)
\end{aligned}
$$

where $t=1, \ldots, n, \boldsymbol{B} n p\left(C l_{t}^{\geq}\right)$and $\boldsymbol{B} n p\left(C l_{t}^{<}\right)$are $P$-doubtful regions. $\underline{P}\left(C l_{t}^{\geq}\right)$is defined by requiring that the largest union of $P$-dominating sets should be included in $C l_{t}^{\geq} . \bar{P}\left(C l_{t}^{\geq}\right)$is defined by requiring that the smallest union of $P$-dominating sets should contain all elements of $C l_{t}^{2}$. These two approximations present the proper and possible assignments of objects into $\mathrm{Cl}_{t}^{2}$ respectively. The objects belonging to the possible but not proper assignment are categorized as doubtful. The quality of DRSA can be explained with the coverage rate defined by Pawlak $(1997,2002)$ and Greco et al. $(2000,2001)$. There are two typical coverage rates $(C R)$ for the upward union $C l_{t}^{2}$ and the downward union $C l_{t}^{<}$, which are formulated as:

$C R\left(C l_{t}^{2}\right)=\frac{\left|\underline{P}\left(C l_{t}^{2}\right)\right|}{\left|C l_{t}^{\Sigma}\right|}$ and $C R\left(C l_{t}^{<}\right)=\frac{\left|\underline{P}\left(C l_{t}^{<}\right)\right|}{\left|C l_{t}^{<}\right|}$. The symbol $C R$ is used to express "the probability of objects in the $P$-lower approximation relatively belonging to the corresponding union of decision classes." The possible assignment can be explained by the accuracy rate. Two typical accuracy rates $(\alpha)$ are listed as:

$\alpha\left(C l_{t}^{\geq}\right)=\frac{\left|\underline{P}\left(C l_{t}^{\geq}\right)\right|}{\left|\bar{P}\left(C l_{t}^{\geq}\right)\right|}=\frac{\left|\underline{P}\left(C l_{t}^{\geq}\right)\right|}{|U|-\left|\underline{P}\left(C l_{t}^{<}\right)\right|}$and $\alpha\left(C l_{t}^{<}\right)=\frac{\left|\underline{P}\left(C l_{t}^{<}\right)\right|}{\left|\bar{P}\left(C l_{t}^{<}\right)\right|}=\frac{\left|\underline{P}\left(C l_{t}^{<}\right)\right|}{|U|-\left|\underline{P}\left(C l_{t}^{\geq}\right)\right|}$. The symbol $\alpha$ is used to present "a ratio of the cardinalities of $P$-lower approximation to those of $P$-upper approximation, i.e., the degree of the properly classified approximation relative to the possibly classified approximation." The ratios can be operated into new ratio in mathematics, proposed by Saaty (2001). 
The DEMATEL technique provides a comprehensive method for building and analyzing a structural model involving effective relationships among complex perspectives (Wu and Lee, 2007) and constructing the correlations between criteria to build a network relationship map, NRM (Tzeng et al., 2007; Huang et al., 2007; Ou Yang et al., 2008). This technique has been successfully applied for a variety of purposes such as creating marketing strategies and dealing with safety problems (Chiu et al., 2006; Liou et al., 2007). In addition, it has helped to develop the competencies of global managers (Wu and Lee, 2007), enabled socially responsible investment (Tsai et al., 2009) and assisted with cost evaluation in the hotel industry (Tsai et al., 2010). The followings present the hybrid of DRSA and DEMATEL to analyze national competitiveness in 2012.

\section{Induction based on HDD (Hybrid of DRSA and DEMATEL)}

\subsection{Dataset}

This research adopts the WCY dataset 2012 containing 59 nations, 4 consolidate factors, and 20 criteria.

\subsection{The hybrid of DRSA and DEMATEL (HDD)}

The hybrid of DRSA and DEMATEL starts from an information system of DRSA to a matrix of the criteria interdependence as the followings. From now on the objects in DRSA are termed as nations.

Proposition 1: Information system of DRSA

$$
D R S A=(U, Q, f, V) \quad \text { where } \quad U=\left\{y_{k} \mid k=1, \ldots, n\right\}, \quad Q=\left\{q_{1}, q_{2}, \ldots, q_{m}\right\}, \quad \text { and } \quad f: U \times Q \rightarrow V,
$$

$V_{Q}=\left(V_{q 1}, V_{q 2}, \ldots, V_{q m}\right)$. This proposition transforms sets into an information system.

Proposition 2: Preference orders

$r_{x j} \succsim r_{z j} \Leftrightarrow f\left(x, q_{j}\right) \geq f\left(z, q_{j}\right), \forall x, z \in U$ where $f$ is a function that maps a criterion to a preference value for a nation. For instance, $r_{x j}$ and $r_{z j}$ are preference values of nation $x$ and $z$ with respect to $q_{j}$.

Proposition 3: A conditional dependent rules $q_{j, t^{\prime}}^{2} \rightarrow q_{i, t}^{2}$

$q_{j, t^{\prime}}^{\geq} \rightarrow q_{i, t}^{\geq}$represents how a criterion $q_{j}$ conditionally depends on the top $t$ positions of $q_{i}$ where $q_{j, t^{\prime}}^{\geq}$is a set of nations within the top $t^{\prime}$ positions with respect to $q_{j}, q_{i, t}^{2}$ is a set of nations within the top $t$ positions with respect to $q_{i}$, and $t^{\prime}$ and $t$ are rank places. In this research $t$ is set as $10^{\text {th }}$ but $t^{\prime} \in\{1,2, \ldots, n\}$. The approximations related to a rule can be conceptualized as Fig. 1. $\underline{P}\left(q_{i, t}^{\geq}\right)$is the lower approximation containing the boundary nation $\underline{x}$ and nations at least as good as $\underline{x}$ in all considered criteria in $P . \bar{P}\left(q_{i, t}^{\geq}\right)$is the upper approximation containing the boundary nation $\bar{x}$ and nation at least as good as $\bar{x}$ in all considered criteria. $\left(q_{j, t^{\prime}}^{\geq}=\bigcup_{s \geq t^{\prime}} q_{j, s}\right)$ contains nations ranked in at least $t^{\prime}$ with respect to criterion $q_{j}$. So is $\left(q_{i, t}^{\geq}=\bigcup_{s \geq t} q_{i, s}\right)$.

Proposition 4: Approximations of $q_{j, t^{\prime}}^{\geq} \rightarrow q_{i, t}^{\geq} . \underline{P}_{j i, t}=\left\{x \in q_{i, t}^{\geq}, D_{P}^{+}(\underline{x}) \subseteq q_{i, t}^{\geq}, P=\left\{q_{j}\right\}\right\}, \bar{P}_{j i, t}=\bigcup_{x \in U} D_{P}^{+}(\bar{x})$, $B n p_{j i, t}=\bar{P}_{j i, t}-\underline{P}_{j i, t}$, and $P=\left\{q_{j}\right\}$. The boundary nations are presented as the slash lines.

Proposition 5: The accurate coverage rate (ACR) of $q_{j, t^{\prime}}^{\geq} \rightarrow q_{i, t}^{\geq}$is formulated as $g_{j i}^{\prime}=g\left(q_{j, t^{\prime}}^{\geq} \rightarrow q_{i, t}^{\geq}\right)$ which is a unique value to present the degree that $q_{j}$ conditionally depends on the top $t$ positions of $q_{i}$, $0 \leq g_{j i}^{\prime} \leq 1$. It is formulated as Model I.

Model I: $\operatorname{Max} g_{j i}^{\prime}=C R_{j i} \times \alpha_{j i}$ 
s.t. $\underline{P}_{j i, t}=D_{P}^{+}(\underline{x}), \bar{P}_{j i, t}=D_{P}^{+}(\bar{x}) ; C R_{j i}=\frac{\left|\underline{P}_{j i, t}\right|}{\left|q_{i, t}^{\geq}\right|}, \alpha_{j i}=\frac{\left|\underline{P}_{j i, t}\right|}{\left|\bar{P}\left(q_{i, t}^{\geq}\right)\right|}$

where $C R_{j i}$ represents a coverage rate of $q_{j, t^{\prime}}^{2} \rightarrow q_{i, t}^{2} . \alpha_{j i}$ represents an accuracy rate [27, 28]. Both the coverage and accuracy rates can be integrated together through a ratios operation proposed by Saaty [29]. One point to note is that the rank of $\underline{x}$ higher than or equal to the rank of $\bar{x}$ with respect to $q_{j}$.

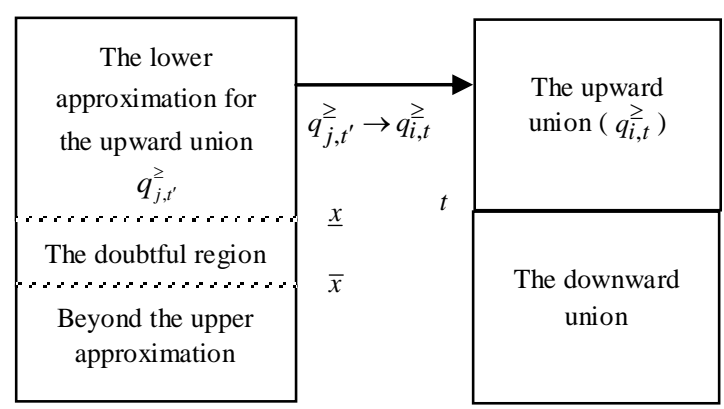

Fig. 1 A dominating rule for approximations

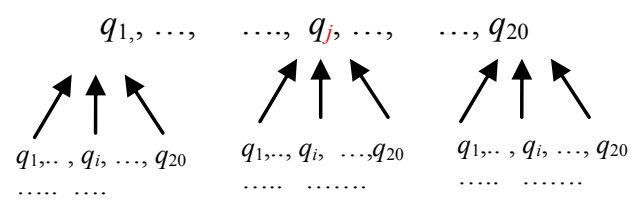

Fig. 2 The structure of criteria interdependence

\subsection{The structure of criteria interdependence}

The structure of criteria interdependence can be constructed by induction rules of Proposition 3 by considering the endless interdependence among criteria as Fig. 2. Each arrow means a conditional dependence of one criterion on another criterion. Technically, the conditional dependence represents a membership degree of a criterion close to another criterion, i.e., an influence. On the other direction of the influence is the sensitivity of a criterion from another criterion. The interdependence rules can be constructed as the following propositions.

Proposition 6: An interdependence rule $\left[q_{j, t^{\prime}}^{2} \rightarrow q_{i, t}^{\geq}\right] \wedge\left[q_{i, t^{\prime}}^{2} \rightarrow q_{k, t}^{\geq}\right] \rightarrow\left[q_{j, t^{\prime}}^{2} \rightarrow q_{k, t}^{\geq}\right] \cong R_{j i} \wedge R_{i k} \rightarrow R_{j k}$

The rule has approximations $\underline{P}_{j i k, t}=\left\{x \in q_{k, t}^{\geq}, D_{P}^{+}(\underline{x}) \subseteq q_{k, t}^{\geq}, P=\left\{q_{j}, q_{i}\right\}\right\}, \bar{P}_{j i k, t}=U-\underline{P}_{j i k, t}$, where $D_{P}^{+}(\underline{x})$ performs like $\quad D_{q_{j}}^{+}(\underline{x}) \wedge D_{q_{i}}^{+}(\underline{x})$. Its qualities include $\quad C R_{j i k}=\frac{\left|\underline{P}_{j i k, t}\right|}{\left|q_{k, t}^{z}\right|}, \quad \alpha_{j i k}=\frac{\left|\underline{P}_{j i k, t}\right|}{\left|\bar{P}_{j i k, t}\right|}, \quad$ and $g_{j i k}^{\prime}=\min \left\{\max \left\{C R_{j i l} \times \alpha_{j i k}\right\}, g_{j i}^{\prime}, g_{i k}^{\prime}\right\}$ which restricts the accurate coverage rate as the minimum possibility along the conjunctives.

Proposition 7: The possibility of an interdependence rule is expressed as $\operatorname{Pos}_{j k}=\operatorname{Pos}\left(\left[R_{j k}\right] \cup\left[R_{j i} \wedge R_{i k} \rightarrow R_{j k}\right] \cup \ldots\right)=\max \left(\operatorname{Pos}\left(R_{j k}\right), \operatorname{Pos}\left(R_{j i} \wedge R_{i k} \rightarrow R_{j k}\right), \ldots\right)$

So, $\operatorname{Pos}_{j k}=\operatorname{Max}\left\{g_{j k}^{\prime}, g_{j i k}^{\prime}, g_{j i i^{\prime} k}^{\prime}, \ldots\right\}$ where $\operatorname{Pos}\left(R_{j k}\right)=g_{j k}^{\prime}, \operatorname{Pos}\left(R_{j i k}\right)=g_{j i k}^{\prime}, \ldots$, etc.

Proposition 8: a conjunctive possibility, $g_{j i k}^{\prime}$

$$
\operatorname{Pos}\left(R_{j i} \wedge R_{i k} \rightarrow R_{j k}\right) \leq \min \left\{\operatorname{Pos}\left(R_{j i}\right), \operatorname{Pos}\left(R_{i k}\right)\right\} \text {. So, } g_{j i k}^{\prime} \leq \min \left\{g_{j i}^{\prime}, g_{i k}^{\prime}\right\} \text { and } g_{j i k}^{\prime} \leq \min \left\{g_{j i}^{\prime}, g_{i k}^{\prime}, g_{j k}^{\prime}\right\} \text {. }
$$

\subsection{DEMATEL induction}

A matrix composed of the accurate coverage rates is designed as Ex. (1) by DEMATEL considering the interdependence between any two criteria iteratively.

$G=G^{\prime} \cup\left(G^{\prime} \bullet G^{\prime}\right) \cup\left(G^{\prime} \bullet G^{\prime} \bullet G^{\prime}\right) \cup \ldots$ 


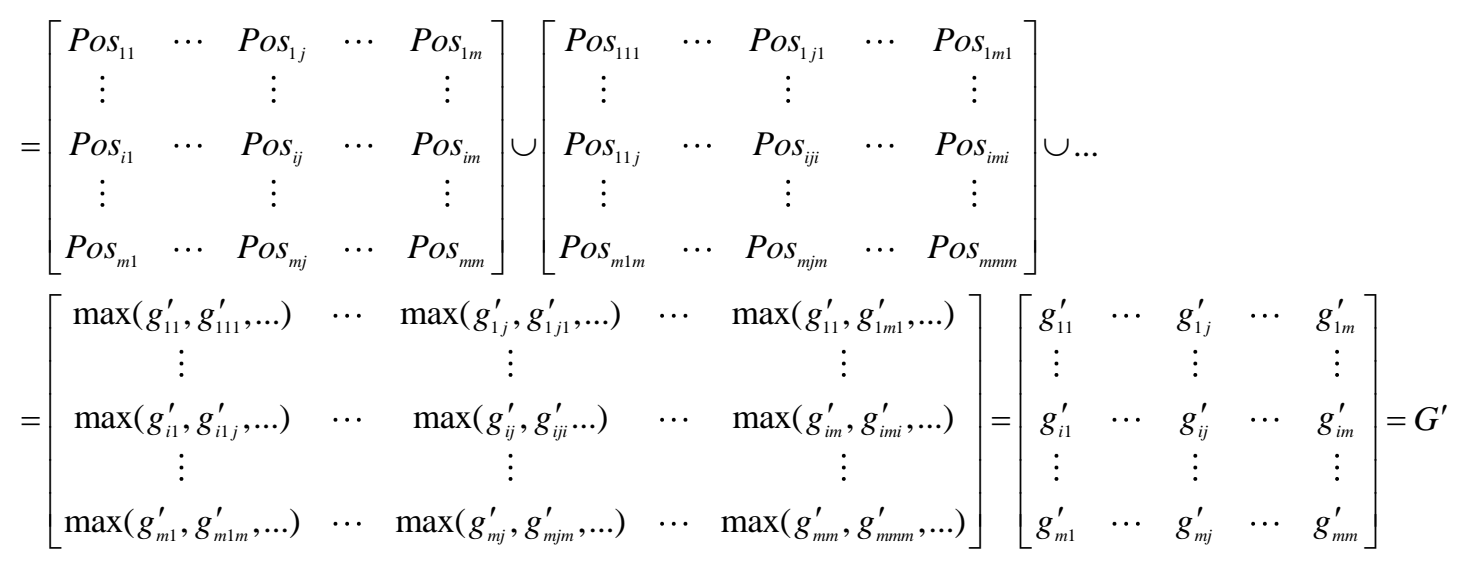

\section{The application results}

The network relationship map (NRM) of the criteria interdependence, $\boldsymbol{G}=\left[g_{j i}^{\prime}\right]$, is constructed to show the influence and sensitivity among criteria. The membership degrees of a criterion to the others means if it changes then the others will change. Its sum is called as the influence. The membership degrees from the others to a criterion means if the others change will cause its change. Its sum is called sensitivity. They are formulated as: Influence vectors: $\boldsymbol{r}=\left(r_{1}, . ., r_{j}, . ., r_{m}\right), r_{j}=\sum_{i=1}^{m} g_{j i}^{\prime} ;$ Sensitivity vectors: $\boldsymbol{d}=\left(d_{1}, . ., d_{j}, . ., d_{m}\right)$, $d_{j}=\sum_{i=1}^{m}\left(g_{j i}^{\prime}\right)^{T}=\sum_{i=1}^{m} g_{i j}^{\prime}$ where $r_{j}$ represents the influence of $q_{j}$ in the others, $d_{i}$ represents the sensitivity of $q_{j}$ to the others. NRM is then illustrated with vertical influence and horizontal sensitivity.



Fig. 3 The resulted NRM

As users can see the criteria, $q_{8}, q_{13}$, and $q_{19}$ have biggest influence and $q_{14}$ has the biggest sensitivity. Apparently, the business efficiency is not only highly sensitive but also influencing. For policy making it needs to consider three aspects, i.e., infrastructure, government, and business efficiency.

\section{Discussion and concluding remarks}

This research finds out the significant criteria of the influence and sensitivity. The resulted Fig. 3 illustrates most criteria have higher influence effects than sensitivity. The institution framework $\left(q_{8}\right)$, nosiness finance $\left(q_{13}\right)$, and management practice $\left(q_{14}\right)$ appear uniquely important due to its sensitivity and influence. Especially, institution framework $\left(q_{8}\right)$ plays a role like the benchmark of competitiveness because it is public and under estimation of WCY. Stakeholders can easily realize the going of nations. Technically, the DEMATEL provides knowledge of criteria interdependence by using the membership degrees to express the influencing and sensitivity for each criterion. Users can easily find out significant criteria in the preferences system.

\section{REFERENCES}

Chiu, Y.J., Chen, H.C., Tzeng, G.H., \& Shyu, J.Z., (2006). Marketing strategy based on customer behavior for the 
LCD-TV, International Journal of Management and Decision Making, Vol.7 No.2/3, pp.143-165.

Fan, T.F., Liu, D.R., \& Tzeng, G.H., (2007). Rough set-based logics for multicriteria decision analysis, European Journal of Operational Research, Vol.82, No.1, pp.340-355.

Greco, S., Matarazzo, B., \& Slowinski, R., (2000). Extension of the rough set approach to multicriteria decision support, INFOR, Vol.38, No.3, pp.161-193.

Greco, S., Matarazzo, B., \& Slowinski, R., (2001). Rough set theory for multicriteria decision analysis, European Journal of Operational Research, Vol.129, No.1, pp.1-47.

Greco, S., Matarazzo, B., \& Slowinski, R., (2002). Rough approximation by dominance relations, International Journal of Intelligent Systems, Vol.17, No.2, pp.153-171.

Huang, C.Y., Shyu, J.Z., \& Tzeng, G.H., (2007). Reconfiguring the innovation policy port- folios for Taiwan's SIP Mall industry, Technovation, Vol.27, No.12, pp.744-765.

Institute for Management Development [IMD], (2012). World Competitiveness Yearbook 2010. Institute Management Development, Lausanne, Switzerland.

Liou, J.J.H., Tzeng, G.H., \& Chang, H.C., (2007). Airline safety measurement using a hybrid model, Air Transport Management Vol.13, No.4, pp.243-249.

Ou Yang, Y.P., Shieh, H.M., Leu, J.D., \& Tzeng, G.H., (2008). A novel hybrid MCDM model combined with DEMATEL and ANP with applications, International Journal of Operations Research, Vol.5, No.3, pp.160-168.

Pawlak, Z., (1997). Rough set approach to knowledge-based decision support, European Journal of Operational Research, Vol.99, pp.48-57.

Pawlak, Z., (2002). Rough set, decision algorithm, and Bayes' theorem, European Journal of Operational Research, Vol.136, No.1, pp.181-189.

Saaty, T. L., (2001). Decision making hierarchies, The Analytic Network Process ( $2^{\text {nd }}$ ed.), RWS Publications.

Tsai, W.H., Chou, W.C., \& Hsu, W., (2009). The sustainability balanced scorecard as a framework for selecting socially responsible investment: an effective MCDM model, Journal of the Operational Research Society, Vol.60, No.10, pp.1396-1410.

Tsai, W.H., Hsu, J.L., Chen, C.H., Lin, W.R., \& Chen, S.P., (2010). An integrated approach for selecting corporate social responsibility programs and costs evaluation in the international tourist hotel, International Journal of Hospitality Management, Vol.29, No.3, pp.385-396.

Tzeng, G.H., Chiang, C.H., \& Li, C.W. (2007). Evaluating intertwined effects in e-learning programs: a novel hybrid MCDM model based on factor analysis and DEMATEL, Expert Systems with Applications, Vol.32, No.4, pp.1028-1044.

U.S. President's Commission on Industrial Competitiveness (1985). Global competition: The new reality, U.S. Government Printing Office, Washington, DC.

The World Economic Forum [WEF] (2003). The global competitiveness report 2003, Oxford University Press for World Economic Forum, New York.

Wu, W.W., \& Lee, Y.T. (2007). Developing global managers' competencies using the fuzzy DEMATEL method, Expert Systems with Applications, Vol.32, No.2, pp.499-507. 